\title{
On the survival of metallicity gradients to major dry-mergers
}

\author{
P. Di Matteo ${ }^{1}$, A. Pipino ${ }^{2}$, M. D. Lehnert ${ }^{1}$, F. Combes $^{3}$, and B. Semelin ${ }^{3}$ \\ 1 Observatoire de Paris, section de Meudon, GEPI, 5 place Jules Janssen, 92195 Meudon, France \\ e-mail: paola.dimatteo@obspm.fr \\ 2 Department of Physics \& Astronomy, University of Southern California, Los Angeles 90089-0484, USA \\ 3 Observatoire de Paris, LERMA, 61 avenue de l'Observatoire, 75014 Paris, France
}

Received 23 January 2009 / Accepted 8 March 2009

\begin{abstract}
Using a large suite of galaxies with a variety of concentrations and metallicity gradients, we study the evolution of non-dissipative ("dry") equal mass mergers. Our purpose in generating these simulations is to explore how the metallicity gradient in dry mergers depends on the structure and metallicity gradients of the galaxies involved in the merger. Specifically, we would like to answer: Could dry mergers lead to metallicity gradients as observed in elliptical galaxies in the local Universe? Do dry mergers always lead to a flattening of the initial (i.e., pre-merger) gradient? From this modeling, we conclude that: The ratio of the remnant and the initial galaxy slopes span a wide range of values, up to values greater than 1 (with values greater than one resulting only when companions have gradients twice the progenitor). For a merger between two ellipticals having identical initial metallicity slopes (i.e., equal companion and galaxy slopes), the metallicity profile of the remnant flattens, with a final gradient about 0.6 times the initial one. Ellipticals can maintain their original pre-merger metallicity gradient if the companion slope is sufficiently steep. The amount of flattening neither depends on the characteristics of the orbit of the progenitors or on their initial concentration. Given the diversity in outcomes of the mergers, we conclude that dry mergers do not violate any observational constraints on the systematic characteristics of metallicity gradients in local ellipticals. In fact, dry mergers may be important within the context of the results of our simulations and may explain the large scatter in gradients for massive ellipticals and the relative paucity of massive ellipticals with no or shallow metallicity gradients.
\end{abstract}

Key words. methods: $N$-body simulations - methods: numerical - galaxies: evolution - galaxies: interaction galaxies: elliptical and lenticular, $\mathrm{cD}$

\section{Introduction}

Recently, we have seen a revision in our understanding of the formation of elliptical galaxies which was motivated by the need to reconcile the apparently "anti-hierarchical" behavior of Active Galactic Nuclei (e.g., Cattaneo \& Bernardi 2003; Hasinger et al. 2005), the evolution of their luminosity function with redshift (e.g., Bundy et al. 2006), as well as the various lines of arguments based on the analysis of their stellar populations (Nelan et al. 2005). In particular, recent results suggest that we need a substantial modification in how we treat the relationship between the baryons and the dark matter in early type galaxies. Pointedly, more massive ellipticals are older and have formed faster than their lower mass counterparts (Thomas et al. 2005). In addition, independent evidence that supports such downsizing is inferred from the systematic characteristics of abundance ratios in elliptical galaxies, namely, the increase of the mean $[\mathrm{Mg} / \mathrm{Fe}]$ in the stellar populations of ellipticals with galaxy mass (Worthey et al. 1992; Matteucci 1994).

Hierarchical modeling, in its most recent incarnations, only partly accounts for the downsizing observed in elliptical galaxies. In practice in such models, significant mass assembly still occurs at late times, but most of the stars have been formed at high redshift in small subunits that merge to grow early type galaxies. The currently preferred mechanism for the assembly of massive spheroids is a sequence of "dry" mergers" (e.g., Khochfar \& Burkert 2003; De Lucia et al. 2006; Cattaneo et al. 2006, 2008). There is evidence that dry mergers might solve some of the outstanding issues related to the growth and evolution of elliptical galaxies. For instance, the brightest ellipticals have central phase-space densities comparable to those of disk galaxies, suggesting that, if mergers are responsible of their build-up, they likely require only a small amount, if any, dissipation (Carlberg 1986). Moreover, dry mergers might explain the strong size evolution of massive red galaxies with redshift (see, e.g., van Dokkum et al. 2008; Khochfar \& Silk 2006) and the formation of slowly rotating ellipticals with boxy isophotes (Naab et al. 2006).

One important aspect of determining whether or not dry mergers are a viable mechanism for explaining the evolution of elliptical galaxies is how various aspects concerning the metal content and distribution changes after a merger event. Pipino \& Matteucci (2008) show that a series of multiple dry mergers (with no associated star-formation) involving buildingblocks that satisfy the $[\mathrm{Mg} / \mathrm{Fe}]$-mass relation cannot fit the mass-metallicity relation and vice-versa (see also Ciotti et al. 1991; Cimatti et al. 2006). A major dry merger (mass ratios

\footnotetext{
1 In this paper dry merger means a pure dissipationless merger of an elliptical stellar systems, i.e., without any gas or significant subsequent star formation.
} 
approximately about one), on the contrary, does not violate these observational constraints if such a merger occurs between galaxies that already obey both the mass $(\sigma)-[\mathrm{Mg} / \mathrm{Fe}]$ and the mass $(\sigma)$-metallicity relations. However, because this mechanism would only operate over a limited range of initial masses, meaning, you cannot have a long series of major mergers to make the most massive ellipticals that initially obeyed the massmetallicity relation, thus this process alone cannot be the underlying physical cause of these trends.

But global trends and relationships amongst ellipticals is not the only constraint available in testing the validity of the dry merger scenario - the structure within individual galaxies can provide further significant constraints. Radial negative metallicity gradients are a common feature in the stellar populations of spheroids (Carollo et al. 1993; Davies et al. 1993). Observations show that the majority of ellipticals have a typical decrease in metallicity of 0.3 dex per decade in radius but with a large scatter (Annibali et al. 2007). Moreover, a positive correlation between the metallicity gradient with the galactic mass has been originally claimed by Carollo et al. (1993), but only for masses lower than $\sim 10^{11} M_{\odot}$. More recently, additional studies have also found a positive correlation of the gradient with mass for a wide range of masses (Ogando et al. 2005; Forbes et al. 2005; Sánchez-Blázquez et al. 2007; Spolaor et al. 2009, but see Kobayashi \& Arimoto 1999; Annibali et al. 2007) and a dependence of elliptical color gradients on the environment, with gentle color gradients for galaxies in dense environments (Ko \& Im 2005).

Steep metallicity gradients are expected from classical dissipative collapse models (e.g., Larson 1974; Carlberg 1985). More modern versions of dissipative collapse starting from (semi-) cosmological boundary/initial conditions (Kawata 1999; Chiosi \& Carraro 2002) can also explain the relation between metallicity gradient slope and stellar mass. The predicted metallicity gradient can be as high as -0.5 dex decade ${ }^{-1}$ in radius, hence galaxies can exhibit a slope steeper than the average. Owing to such a steep predicted slope it has been hypothesised that galaxy mergers and monolithic collapse could be at work together in order to explain the range in slopes (Kobayashi 2004). On the other hand, more realistic models with a detailed treatment of the chemical evolution predict a typical metallicity gradient of -0.25 dex decade $^{-1}$ (Pipino et al. 2008) and also a trend between the gradient and mass. These are just the trends necessary to explain the latest observations (Pipino et al. 2009, in preparation). This finding implies that the assumption that ellipticals are formed through a mixture several channels (including mergers and monolithic collapse, e.g. Kobayashi 2004) is not necessary, but cannot be excluded with current available data.

$\mathrm{N}$-body numerical simulations can be an important tool for understanding the evolution of metallicity gradients in mergers and their remnants. Such simulations are able to account for different environments, mass ratios, and morphologies of the progenitor galaxies. The cosmological simulations by Kobayashi (2004) have shown that a variety of gradients in early-type galaxies can be generated and are mainly due to a difference in merging histories. In such a scenario, gradients are destroyed during mergers, by an amount depending on the mass ratio of the progenitors. Gradients can be regenerated if strong star formation occurs in the central regions - thus requiring sufficient gas dissipation to fuel such star-formation - and they slowly evolve through subsequent gas accretion. These simulations indicate that, in order to reproduce the large scatter observed in metallicity gradients, both gas infall (monolithic collapse) and major mergers must occur. While cosmological simulations can follow
Table 1. Galaxy parameters. Both the stellar and dark mater profiles are represented by Plummer models, having characteristic masses, respectively, given by $M_{\mathrm{B}}$ and $M_{\mathrm{H}}$ and core radii given by $r_{\mathrm{B}}$ and $r_{\mathrm{H}}$.

\begin{tabular}{|c|c|c|c|c|}
\hline & gE0 & gE01 & $\mathrm{gE0m}$ & iE0 \\
\hline$M_{\mathrm{B}}\left[2.3 \times 10^{9} M_{\odot}\right]$ & 70 & 70 & 70 & 35 \\
\hline$M_{\mathrm{H}}\left[2.3 \times 10^{9} M_{\odot}\right]$ & 30 & 30 & 30 & 15 \\
\hline$r_{\mathrm{B}}[\mathrm{kpc}]$ & 4.0 & 6.0 & 2.0 & 2.8 \\
\hline$r_{\mathrm{H}}[\mathrm{kpc}]$ & 7.0 & 7.0 & 7.0 & 5.0 \\
\hline$N_{\text {star }}$ & 80000 & 80000 & 80000 & 40000 \\
\hline$N_{\text {DM }}$ & 40000 & 40000 & 40000 & 20000 \\
\hline
\end{tabular}

Table 2. Orbital parameters.

\begin{tabular}{ccccc}
\hline \hline Orbit id & $\begin{array}{c}r_{\text {ini }} \\
{[\mathrm{kpc}]}\end{array}$ & $\begin{array}{c}v_{\text {ini }} \\
{\left[100 \mathrm{~km} \mathrm{~s}^{-1}\right]}\end{array}$ & $\begin{array}{c}L^{a} \\
{\left[10^{2} \mathrm{~km} \mathrm{~s}^{-1} \mathrm{kpc}\right]}\end{array}$ & $\begin{array}{c}E^{b} \\
{\left[10^{4} \mathrm{~km}^{2} \mathrm{~s}^{-2}\right]}\end{array}$ \\
\hline 01 & 100. & 2.0 & 57.0 & 0. \\
02 & 100. & 3.0 & 59.0. & 2.5 \\
05 & 100. & 2.0 & 80.0 & 0. \\
13 & 100. & 0.0 & 0.0 & -2. \\
14 & 70.0 & 1.5 & 41.3 & -0.66 \\
15 & 70.0 & 1.6 & 41.3 & -1.57 \\
\hline
\end{tabular}

${ }^{a}$ It is the absolute value of the angular momentum of the unit mass, i.e., $L=|\boldsymbol{r} \times \boldsymbol{v}|{ }^{b}$ It is the total energy of the relative motion, i.e., $E=v^{2} / 2-G\left(m_{1}+m_{2}\right) / r$, with $m_{1}=m_{2}=2.3 \times 10^{11} M_{\odot}$.

a number of different merging histories (from the coalescence of small gas-rich subunits to major dry or wet mergers), it is also important to understand the role that each of these processes play in reshaping any initial metallicity profile.

While a limited number of studies have investigated the evolution of metallicity gradients in gas-rich mergers (Mihos \& Hernquist 1994; Bekki \& Shioya 1999), taking into account both dissipative processes and the impact of star formation, less attention has been devoted to the evolution of metallicity profiles in dry mergers. Early attempts at these types of simulations suggested that metallicity gradients flatten, due to both mixing and core-halo differentiation during the merger (White 1980). However, $N$-body experiments have shown that the initial state of the galaxy is not completely washed out during the coalescence of the systems (van Albada 1982), suggesting that radial abundances may be only moderately reduced by dissipationless mergers (Barnes 1996; Mihos \& Hernquist 1994).

Given the importance of this problem for understanding the evolution of early type galaxies, we wish to investigate the impact of dry mergers on galaxy evolution further through the use of $N$-body simulations of equal-mass elliptical galaxies. From this investigation, we want to understand: 1) if dry mergers always cause the flattening of an initial galaxy gradient; 2) under what conditions does this flattening occur; 3) what is the magnitude of the flattening that is typically produced for a range of initial conditions; and 4) how does this evolution depend on orbital and morphological properties of the interacting systems. To achieve this aim, we simulated a number of equal-mass dry mergers, with a variety of orbital parameters, morphologies, and initial metallicity gradients for each component of the merger. We have chosen to begin investigating equal mass mergers, because they are likely to result in the greatest amount of mixing in phase-space and so, ultimately, they should cause the strongest variations in the initial (i.e. pre-merger) metallicity profiles.

The main characteristic of the models (initial conditions and numerical code used) are briefly described in Sects. 2 and 3, 

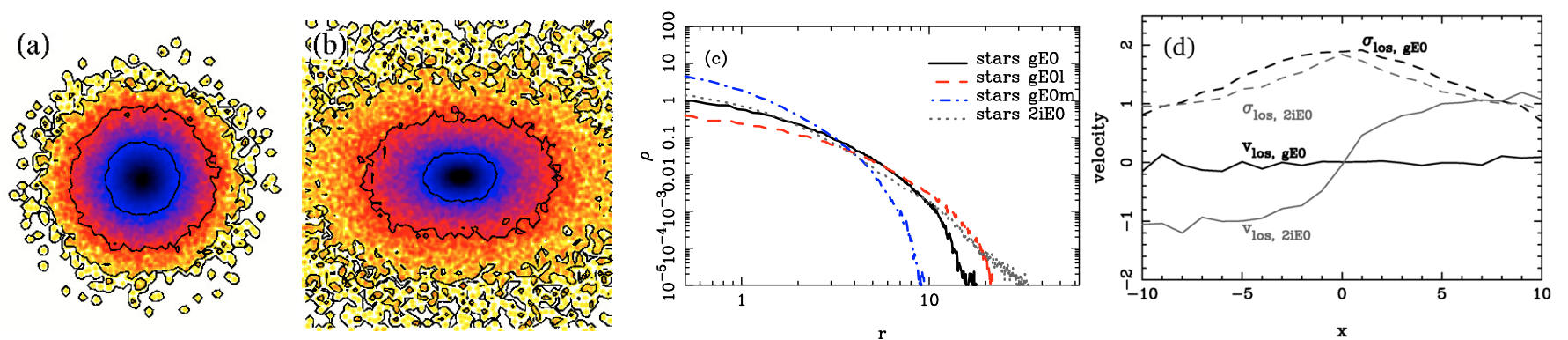

Fig. 1. Initial properties of some galaxy models: a) projected-density map of the gE0 galaxy, after evolving in isolation for 1 Gyr. Contours are equally spaced on a logarithmic scale; b) projected-density map of the 2iE0 galaxy which is the result of a collision between two iE0s elliptical models (see text for details). We used the same scale interval and contours level as in the previous panel; c) volume-density profiles of some galaxy models, as indicated in the legend of the figure; d) line-of-sight velocity and velocity dispersion profiles for the gE0 galaxy model (black solid and black dashed lines respectively) and for the $2 \mathrm{iE} 0$ galaxy merger remnant (gray solid and gray dashed lines respectively).

results are presented and discussed in Sect. 4 and conclusions are drawn in Sect. 5.

\section{The model}

The mergers studied in this paper consist of the coalescence of two equal-mass elliptical galaxies, without any dissipative component. The fiducial case is described in Sect. 4.1. It corresponds to mergers of two giant elliptical E0 (hereafter gE0) galaxies, whose internal parameters are given in Table 1, moving on 6 different orbits ( 2 parabolic, 1 hyperbolic, 1 radial and 2 ellipticals; see Table 2).

To understand to what extent the evolution of the metallicity gradients depends on the galaxy parameters adopted, we also modeled interacting galaxies having a central density 5 times higher and 2.5 times lower than that of the gE0 galaxies (we refer to these systems as gE0m and gE01, respectively; see Table 1 for their morphological parameters). To allow the models to reach an equilibrium and to demonstrate their initial stability, all the modeled galaxies were allowed to evolve in isolation for $1 \mathrm{Gyr}$ before any encounter. This allows us to distinguish internal relaxation phenomena from those due to the merging process itself. These systems represent quite idealized initial conditions: they are completely spherical, showing no rotation in their baryonic component. Observations show, however, a vast range of ellipticities and levels of rotation (see, for example Emsellem et al. 2007). The systematics of the amount of rotation and ellipticity with mass seem to suggest that more massive spheroids are not likely to be the result of dissipative mergers (Carlberg 1986; van Dokkum et al. 2008). Therefore, to test "more realistic" initial conditions, we modeled also mergers between two equal-mass galaxies (hereafter called iE0), having masses equal to 0.5 times that of gE0s. These galaxies were then subsequently merged to form a system (hereafter called 2iE0) whose total mass is equal to that of gE0s, but having initially a different morphology (triaxial instead of spherically symmetric) and a certain amount of rotation (see Table 1 for the initial parameters of the iE0 galaxies and Fig. 1 for some properties of the initial galaxy models). In this way, we were able to, at least partially, account for more complicated merger histories and hopefully more realistic merger components in our simulations.

Each galaxy in the merging pair has been modeled with $N=120000$ particles, distributed among stars and dark matter. We also re-simulated some cases, using a number of particles two and four times higher, attempting to insure that the results presented here do not depend on the numerical resolution adopted in our models.
Table 3. Initial gradients and metallicity profiles for the elliptical galaxies.

\begin{tabular}{ccl}
\hline \hline Gradient id & $\Delta$ & Metallicity profile \\
\hline grad01 & -0.26 & $z(r)=3 z_{\odot} 10^{-0.07 r}$ \\
grad02 & 0.00 & $z(r)=z_{\odot}$ \\
grad03 & -0.13 & $z(r)=3 z_{\odot} 10^{-0.035 r}$ \\
grad04 & -0.13 & $z(r)=1.5 z_{\odot} 10^{-0.035 r}$ \\
grad05 & 0.00 & $z(r)=1.5 z_{\odot}$ \\
grad06 & -0.22 & $z(r)=3 z_{\odot} 10^{-0.06 r}$ \\
grad07 & -0.18 & $z(r)=3 z_{\odot} 10^{-0.05 r}$ \\
grad08 & -0.15 & $z(r)=3 z_{\odot} 10^{-0.04 r}$ \\
grad09 & -0.07 & $z(r)=3 z_{\odot} 10^{-0.02 r}$ \\
grad10 & -0.04 & $z(r)=3 z_{\odot} 10^{-0.01 r}$ \\
grad11 & -0.30 & $z(r)=3 z_{\odot} 10^{-0.08 r}$ \\
grad12 & -0.33 & $z(r)=3 z_{\odot} 10^{-0.09 r}$ \\
grad13 & -0.37 & $z(r)=3 z_{\odot} 10^{-0.10 r}$ \\
grad14 & -0.41 & $z(r)=3 z_{\odot} 10^{-0.11 r}$ \\
grad15 & -0.44 & $z(r)=3 z_{\odot} 10^{-0.12 r}$ \\
\hline
\end{tabular}

Finally, for each of the galaxies involved in the interaction and merger, we have adopted 15 different initial metallicity profiles. These profile were constructed by assigning a metallicity, $Z$, to each star particle in the system that depended exponentially on the distance of the star from the galaxy center (the formal functions are given in Table 3). This is also why the galaxies are allowed to evolve in isolation for $1 \mathrm{Gyr}$. This time allows us to ensure that the metallicity gradient of the isolated galaxy is stable and remains as it was assigned. A large variety of initial central metallicities and gradient slopes are thus studied in this way, ranging from flat profiles (as it is the case of gradient id $=\operatorname{grad02}$ and grad05) to metallicity gradients around -0.3 dex per decade in radius (grad01, grad11, grad12) to quite steep slopes (gradient $\mathrm{id}=\operatorname{grad} 14$, grad15). In this and in the next sections, the metallicity gradient is defined as,

$$
\begin{aligned}
\Delta & =\frac{\Delta \log (Z)}{\Delta \log (r)} \\
& =\frac{\log \left(Z\left(0.1 r_{50}\right)\right)-\log \left(Z\left(r_{50}\right)\right)}{\log \left(0.1 r_{50}\right)-\log \left(r_{50}\right)} \\
& =\log \left(Z\left(r_{50}\right)\right)-\log \left(z\left(0.1 r_{50}\right)\right),
\end{aligned}
$$

where $r_{50}$ is defined as the radius which contains half of the baryonic mass ("baryonic half-mass radius").

In total, we analyzed 28 different simulations of major dry mergers, and, for each interaction, we studied 120 different 


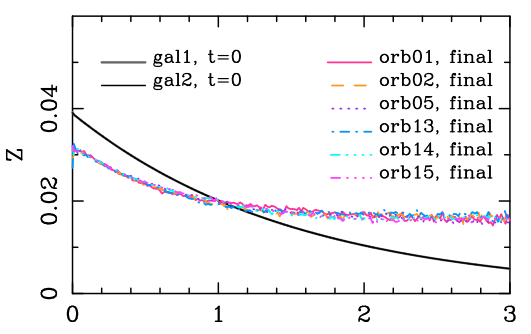

$\mathrm{r} / \mathrm{r}_{50}$

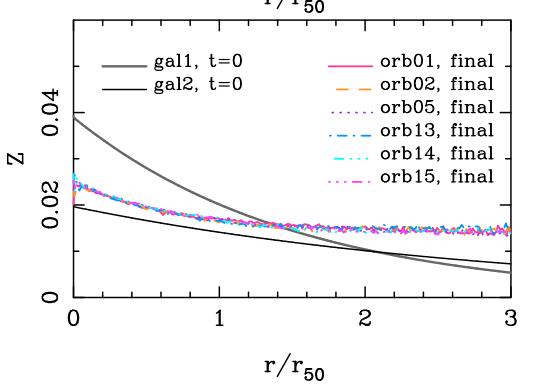

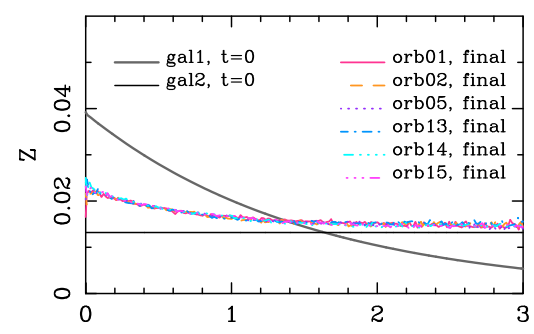

$\mathrm{r} / \mathrm{r}_{50}$

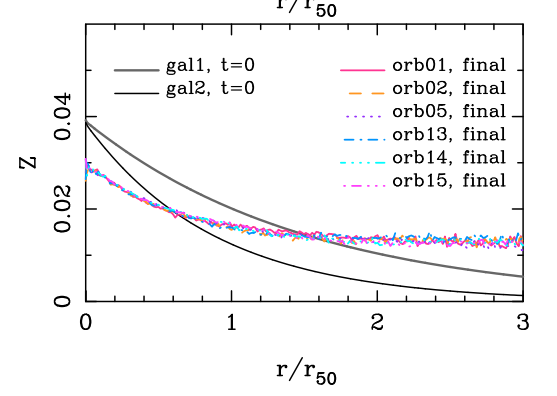

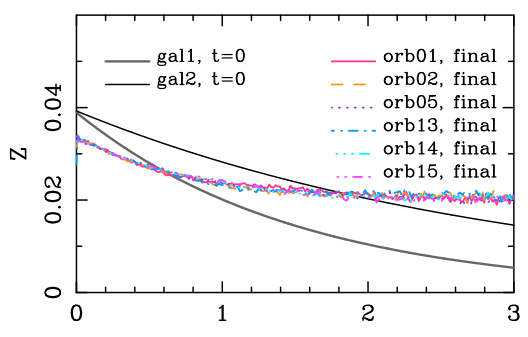

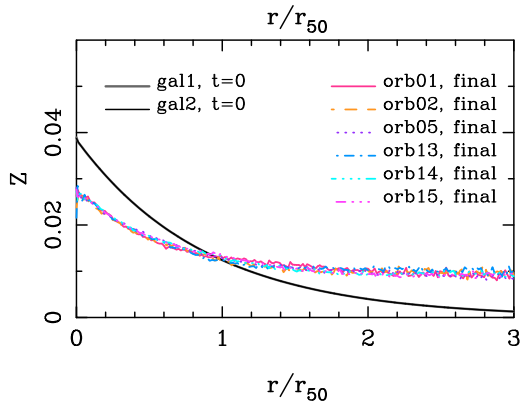

Fig. 2. Evolution of the metallicity profiles in some major (1:1) dry mergers of two gE0 galaxies. Metallicity is shown versus the distance from the galaxy center for the two galaxies before the interaction (black and thick gray solid lines) and for the final remnant for different orbits (see figure legend). The values on the abscissa are shown in units of the half-mass radius $r_{50}$ of the corresponding galaxy. Different panels in the figure correspond to different initial metallicity profiles for the two interacting galaxies: (top-left panel) id = grad01grad01; (top-middle panel $) \mathrm{id}=$ grad01 grad02; $($ top-right panel $) \mathrm{id}=$ grad01 grad03; $($ bottom-left panel $)$ id = grad01 grad04; $($ bottom-middle panel $)$ id = grad01 grad15; (bottom-right panel) id = grad15grad 15 .

combinations of the initial metallicity profiles, corresponding to 3360 different possibilities.

\section{Numerical method}

All the simulations have been run using the Tree-SPH code described in Semelin \& Combes (2002). A further description is given also in Di Matteo et al. (2007) and in Di Matteo et al. (2008). The code uses a hierarchical tree method (Barnes \& Hut 1986) to evaluate gravitational forces and a smoothed particle hydrodynamics method to simulate the evolution of gas Lucy (1977); Gingold \& Monaghan (1982). Since the work presented here only investigates dry-mergers, only the part of the code evaluating the gravitational forces acting on the systems has been used.

Gravitational forces are calculated using a tolerance parameter $\theta=0.7$ and includes orders up to the quadrupole term in a multiple expansion. A Plummer potential is used to soften the gravity at small scales, with constant softening lengths of $\epsilon=280 \mathrm{pc}$ for all particles. The equations of motion are integrated using a leapfrog algorithm with a fixed time step of 0.5 Myr.

\section{Results and discussion}

\subsection{Evolution of metallicity profiles in major dry mergers}

The evolution of some of the metallicity profiles during major non-dissipative mergers are shown in Fig. 2. In this plot the initial morphologies of the two interacting galaxies are always the same, while the orbits and initial metallicity gradients have been varied. In some cases, as for the top-left and bottom-right panels, the two interacting galaxies have initially the same profile. This situation of having identical initial equal metallicity profiles is the case studied by White (1980): in this cases, the profile flattens, due to the redistribution of stars in the systems induced by the merging process. Indeed, one can clearly see from the final profile that the flattening is due to both the decrease of the central metallicity and to the contemporaneous increase of the metallicity in the outer regions. Note also that the evolution of the profile does not seem to be very sensitive to the orbital initial conditions: orbits with different initial energies and angular momenta produce very similar final profiles, at least in the region inside the remnant half-mass radius. This happens because mixing and phase-space density evolution are independent on the orbital parameters, as discussed in Appendix A.

Figure 2 also shows some examples of the two galaxies having different initial metallicity gradients and distributions ("mixed"). As discussed in the Introduction, observations (see, for example Ogando et al. 2005; Spolaor et al. 2009) show that early-type galaxies in the local Universe have a wide dispersion in their metallicity gradients, particularly at the high end of the distribution of masses. This means that dry mergers between galaxies with different metallicity gradients should be common locally. Of course, this is not true if there is some sort of regularity in or correlation between the metallicity gradients as a function of local galaxy density, for which yet there is no clear evidence (see Sánchez-Blázquez et al. 2006; Clemens et al. 2009). But if "mixed" mergers are common (as seems likely), then it is interesting to understand what the resulting gradient is and how it depends on the initial profiles and orbital parameters of the progenitors. By examining the plots (Fig. 2) and analyzing the simulations, the resulting gradients in mixed mergers can lie in between those of the progenitors: the merging of galaxies with a flat and steep gradient $(\Delta=-0.26)$ produce a remnant having a slope of about -0.09 which is lower than the steepest progenitor gradient but certainly not flat as in the companion galaxy. This implies that for a given progenitor galaxy with an initial metallicity gradient, major dry mergers do not necessarily lead to a flattening of the initial galaxy slope, but the outcome depends greatly on the slope of the companion galaxy. An early-type elliptical galaxy merging with an other having the same initial metallicity profile will have a final slope lower than the initial 


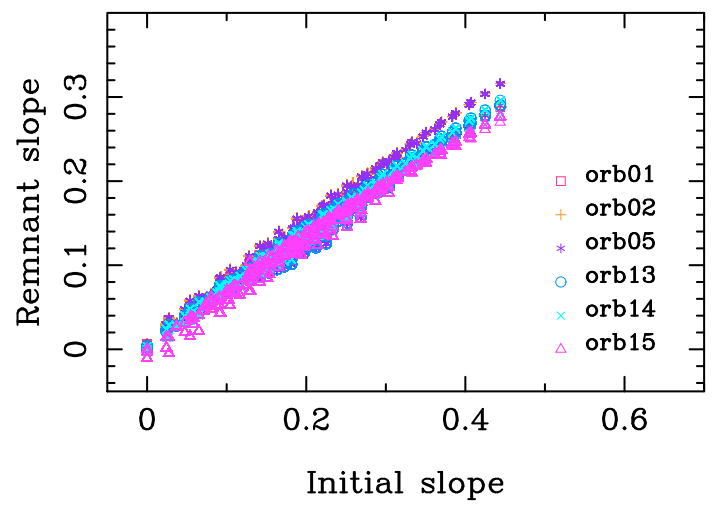

Fig. 3. Absolute values of the initial and final metallicity slopes for gE0-gE0 mergers. Different orbits are indicated by different symbols, as explained in the legend. The initial slope has been evaluated by superposing the initial metallicity profiles of the two progenitor galaxies.

one - the amount of flattening is evaluated in Sect. 4.3 - but if it merges with a companion having a sufficiently steep profile, the final slope can be the same or steeper than the initial one.

Comparing the superposition of the initial metallicity profiles of the two progenitor galaxies (i.e. $Z_{1}(r)+Z_{2}(r)$ ) with the remnant profile, we find that, on average, the final metallicity gradient is a factor $0.6-0.7$ flatter than the initial one, with only a small dispersion around the average (see Fig. 3).

\subsection{Core-halo differentiation and phase-space mixing}

In Sect. 4.1, we showed that the metallicity gradient of the final merger remnant depends on the initial gradients of the progenitor galaxies. Here we present more details about the mechanisms causing the evolution of these profiles. Importantly, we note that the metallicity profiles given in Fig. 2 are shown in units of the stellar half mass radii $r_{50}$ of the progenitors and remnant. Thus, a variation in the half-mass radii of the remnant with respect to that of the progenitor galaxies, without any variation in the $Z(r)$ profile, should be sufficient, in principle, to change the final metallicity profile.

Indeed, as shown for gE0-gE0 mergers in the left panel of Fig. 4, the half-mass radius changes with time, at each pericenter passage and mostly during the final phases of coalescence of the two systems $\left(t / t_{\text {merging }} \simeq 1\right)$, with the final value being about $50 \%$ greater than the pre-interaction value. Note also that outer parts of the systems (those outside $r_{50}$ ) show the strongest evolution. Indeed, the tidal shocks increase the radius $r_{90}$, containing $90 \%$ of the stellar mass by more than a factor of 3. On the contrary, the inner radii show only modest change after the merger. Moreover, the evolution with radius also has a behavior that depends on the orbital geometry of the progenitors. The dynamical evolution of the outer radii is affected by the orbital geometry while that of the inner regions is largely independent of the orbital parameters.

As first shown by White (1980), it is possible to separate the effects due to structural changes from those arising from mixing by showing the metallicity profiles as a function of the enclosed mass rather than as a function of some physical coordinates. If the evolution of the metallicity gradient was only due to corehalo differentiation, then the initial and final profiles in enclosed mass would be identical. However, a clear flattening occurs during the merger (see Fig. 4, middle and right panels), indicating that mixing must contribute to the evolution of the metallicity gradient. In our simulations, this flattening is more pronounced than that found by White (1980). This difference could be due to his choice of concentrating on merger remnants which showed density profiles and velocity structures as close as possible to that of the progenitors (see discussion in White 1980). In addition, the process of mixing does not seem to depend on the initial orbital geometry but it is generic to the merging process itself and leads to rather similar metallicity gradients. This implies that mixing is mostly driven by the progenitor structure that (at least partially) survives the merging process (see also White 1980; Barnes 1996; Valluri et al. 2007). As a consequence, our simulations indicate that it is not possible to disentangle the orbits of the parent galaxies by using the metallicity distribution of the remnant if the analysis is restricted to the central region of the galaxy (i.e., inside the half-mass radius).

\subsection{Metallicity gradients in major dry mergers: flattening or steepening?}

After describing how metallicity profiles evolve during a merger and the physical processes which determine this evolution, we now want to quantify the change in the radial profiles themselves as a function of the initial metallicity distribution.

In Table 4, we tabulate the initial and final metallicity gradients for simulations of two gE0 galaxies with a specific orbital geometry $(\mathrm{id}=01)$ but for a range of metallicity gradients. To generalize this, Fig. 5 shows the absolute values of the initial and final gradients from the simulations for all the galaxy morphologies and orbits. For a given initial metallicity gradient there are a range of final gradients, depending mainly on the profile of the companion galaxy. The initial galaxy models span a wide range in metallicity slopes, $0.0-0.7$, while the remnant slope only spans about $0.0-0.4$. Also, a close inspection of Table 4 tells us that the final slope is always flatter than the maximum between the parent and his companion slopes. In many cases, the final slope is lower than the mimimum between the parent and his companion slopes. Note that, as suggested previously, galaxies having the same morphologies have very similar final slopes, independent of their orbital geometry. This is the case regardless of the initial morphologies. In addition, the most concentrated galaxies $(\mathrm{gE0m})$ have the smallest range of gradients and generally smaller gradients for the same initial conditions while galaxies with low concentrations (e.g., gE0l) generally have larger slopes and exhibit a wider range of slopes. This is due to the fact that we have assigned to each galaxy the metallicity profiles following a simple $Z=Z(r)$ law where $r$ is the physical distance from the galaxy center of mass. That is, the initial metallicity profiles have not been normalized in anyway to the galaxy half-mass radii, while the metallicity gradients, according to Eq. (1), are evaluated using them.

A comparison of the evolution of the metallicity profiles for galaxies with different initial concentration is shown in Fig. 6. In the left panel, the initial metallicity profile is shown as a function of $r$, the distance from the center, for the gE01, gE0 and gE0m galaxy. This initial profile is obviously the same for the three galaxies, accordingly to our choice. The final profile of the remnant galaxy, in turn, depends on the initial galaxy density, in the sense that the more concentrated the galaxy is, the higher is the value of $Z(r)$, for any given $r$. This is simply a consequence of the fact that the more concentrated the galaxy is, the more metal rich it is too, according to the way the initial metallicity profile has been assigned. When normalizing to the half-mass radius (right panel in Fig. 6), in turn, the initial profiles are obviously different, as well as the final ones, but interestingly the amount of flattening of the final gradients with respect to the initial ones are very similar. If the two galaxies have initially 

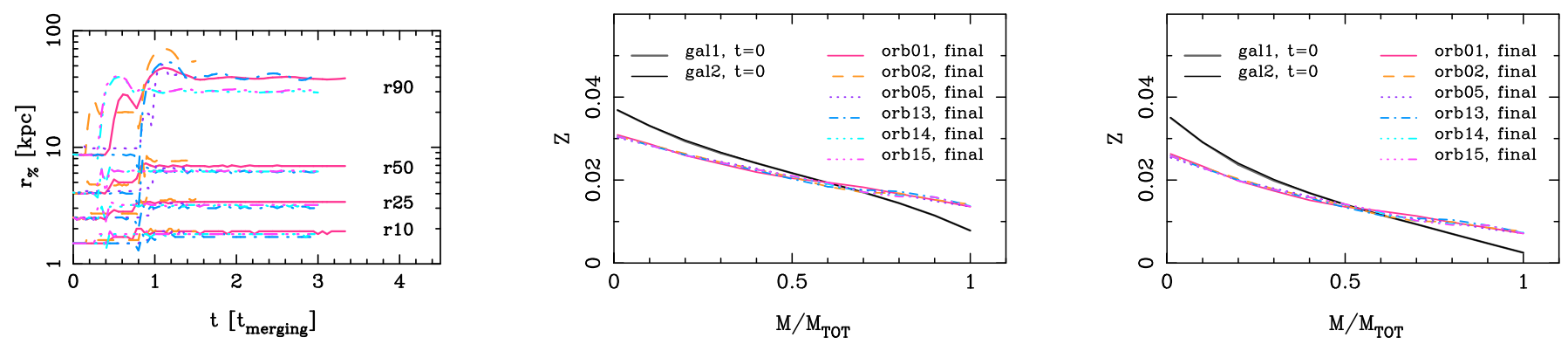

Fig. 4. Core-halo differentiation and mixing in some of major gE0-gE0 mergers modeled for this study. (left) Shows the evolution with time of the Lagrangian radii containing $10 \%, 25 \%, 50 \%, 75 \%$, and $90 \%$ of the total stellar mass. For simplicity, the radii are shown only for stars belonging to one of the two galaxies (the behavior is the same for the companion). The time axis is in units relative to the merging time. Mergers with a variety of orbital characteristics are shown, as indicated into the figure legend. (Middle and right) Metallicity profiles for some gE0-gE0 mergers, as a function of the fractional mass contained. The panels correspond to different metallicity profiles: id= grad01 grad01 ( middle panel); $\mathrm{id}=\operatorname{grad} 15 \mathrm{grad} 15$ (right panel). Metallicity is shown for the two galaxies before the interaction (black and thick gray solid lines) and for the final remnant.

identical metallicity profiles, as in the case shown in Fig. 6, the final metallicity gradient will have a slope equal to $0.6-0.7$ times the initial one, with no clear correlation with the initial central density of the galaxy.

Figure 5 shows that a range of initial gradients gives rise to a variety of remnant slopes, this depending, of course, on the slope of the companion galaxy, as well as on the initial concentration. What is the relationship between the remnant and initial galaxy slopes? This is shown in Fig. 7. The relation between the initial and remnant gradients has generally a small scatter about a mean relation, with higher dispersion for the high ratios. This figure basically summarize the results of this work which implies:

- the ratio of the remnant and the initial galaxy slopes span a wide range of values, up to values greater than 1 ;

- for a merger between two ellipticals having identical initial metallicity slope (i.e., companion slope $=$ galaxy slope), the metallicity profile of the remnant flattens, with a final gradient about 0.6 times the initial one;

- remnants can have metallicity gradients greater than that of the progenitor elliptical galaxy. Our simulations show that this happens every time the companion has a slope two times greater than the parent;

- ellipticals can maintain their original pre-merger metallicity gradient if the companion slope is sufficiently steep;

- the final remnant gradient does not depend on the orbits of the progenitor ellipticals;

- while the final gradient does depend on the initial concentration of the progenitors, the amount of flattening (i.e. remnant slope/progenitor slope) does not.

\subsection{The role of dissipationless mergers in the evolution of elliptical galaxies}

Do these results in any way suggest that equal mass dry mergers might be inconsistent with the metallicity gradients observed in local ellipticals? The short answer is no. There are no systematic trends in magnitude of metallicity gradients in ellipticals save one, metallicity gradients generally become steeper with increasing mass, but with a large scatter (Ogando et al. 2005; Spolaor et al. 2009). Our models show that, as long as the metallicity gradient of the companion is not more than twice that of the parent, the remnant will have a shallower gradient. Thus one can imagine that the build up of the most massive ellipticals is through a series of dry mergers whereby the gradient gradually softens leading to a trend, albeit with large scatter, between mass and metallicity gradient. But if there are several mergers in sequence with long times between then, the metallicity gradients would increase and decrease rather randomly, thus leading to a situation where there are few systematic trends between the characteristics of the metallicity gradient and structure properties of ellipticals. This is precisely shown in Fig. 8, where the evolution of an initial metallicity gradient, due to recursive dry mergers, is followed. If dry mergers can occur among ellipticals having different gradients, randomly distributed in the shaded area $^{2}$ shown in the plot, a sequence of such events does not lead to a clear trend (for example, the more massive is the galaxy, the shallower its profile). In turn, a sort of random walk, with increasing and decreasing slopes, seems to be the general outcome (upper panel in Fig. 8), unless at each merger epoch, a given galaxy is allowed to merge only with companions having the identical gradient (lower panel in Fig. 8). Because of the stochastic nature of the process of dry mergers, it is difficult to gauge the frequency, and therefore the importance of this process in galaxies. While, most likely, the merger of two similar ellipticals would lead to a lessening of the metallicity gradient, the final product depends on the concentration of the individual galaxies and their initial gradients. As the gradient of the parent galaxy because flatter and flatter, it then becomes likely that another dry merger will actually increase its metallicity gradient. If some of the mergers are not completely dissipationless, then this will also tend to increase the gradient. However, the relative mass of gas that forms stars during any gas accretion or merger must be small. If the fraction of mass forming stars were large or did so over extended periods of time, then this process would violate the well-known enhancement in $[\alpha / \mathrm{Fe}]$ in ellipticals, especially the most massive ones. Therefore, any enhancement in the gradient due to star-formation must be small and the influence of the dissipationless component must be dominant in determining the final metallicity gradients in massive elliptical galaxies.

One of the obvious outcomes of all of this is that if ellipticals at all masses were formed with the same gradient then we would expect the scatter in the metallicity gradient as a function of mass to increase with both time and mass. This is simply because our simulations show that metallicity gradients that are initially steep will most likely soften. This will continue up to the point where the gradient is shallow enough to make it more likely that the merger will be with a galaxy that has a slope steep enough (by a factor of 2) to actually increase the gradient in the

\footnotetext{
2 This area encloses the negative metallicity slopes found by Ogando et al. (2005), and as shown in their Fig. 2.
} 
Table 4. Initial and final metallicity gradients for the fiducial 1:1 dry mergers.

\begin{tabular}{|c|c|c|c|c|c|c|c|}
\hline Run type & $\begin{array}{c}\text { Initial slope } \\
\text { (Galaxy 1) }\end{array}$ & $\begin{array}{c}\text { Initial slope } \\
\text { (Galaxy 2) }\end{array}$ & $\begin{array}{l}\text { Final slope } \\
\text { (Remnant) }\end{array}$ & Run type & $\begin{array}{c}\text { Initial slope } \\
\text { (Galaxy 1) }\end{array}$ & $\begin{array}{c}\text { Initial slope } \\
\text { (Galaxy 2) }\end{array}$ & $\begin{array}{l}\text { Final slope } \\
\text { (Remnant) }\end{array}$ \\
\hline grad01 grad01 & -0.26 & -0.26 & -0.17 & grad05grad11 & 0.00 & -0.30 & -0.11 \\
\hline $\operatorname{grad} 01 \operatorname{grad} 02$ & -0.26 & 0.00 & -0.09 & $\operatorname{grad} 05 \operatorname{grad} 12$ & 0.00 & -0.33 & -0.12 \\
\hline $\operatorname{grad} 01 \operatorname{grad} 03$ & -0.26 & -0.13 & -0.13 & $\operatorname{grad} 05 \operatorname{grad} 13$ & 0.00 & -0.37 & -0.13 \\
\hline grad01 grad04 & -0.26 & -0.13 & -0.13 & grad05grad14 & 0.00 & -0.41 & -0.13 \\
\hline grad01grad05 & -0.26 & 0.00 & -0.08 & $\operatorname{grad} 05 \operatorname{grad} 15$ & 0.00 & -0.44 & -0.14 \\
\hline grad01 grad06 & -0.26 & -0.22 & -0.16 & grad06grad06 & -0.22 & -0.22 & -0.15 \\
\hline grad01grad07 & -0.26 & -0.18 & -0.15 & grad06grad07 & -0.22 & -0.18 & -0.14 \\
\hline grad01grad08 & -0.26 & -0.15 & -0.14 & grad06grad08 & -0.22 & -0.15 & -0.13 \\
\hline grad01 grad09 & -0.26 & -0.07 & -0.11 & grad06grad09 & -0.22 & -0.07 & -0.10 \\
\hline grad01 grad10 & -0.26 & -0.04 & -0.09 & $\operatorname{grad} 06 \operatorname{grad} 10$ & -0.22 & -0.04 & -0.08 \\
\hline $\operatorname{grad} 01 \operatorname{grad} 11$ & -0.26 & -0.30 & -0.18 & grad06grad11 & -0.22 & -0.30 & -0.17 \\
\hline grad01grad12 & -0.26 & -0.33 & -0.19 & $\operatorname{grad} 06 \operatorname{grad} 12$ & -0.22 & -0.33 & -0.18 \\
\hline grad01grad13 & -0.26 & -0.37 & -0.20 & grad06grad13 & -0.22 & -0.37 & -0.18 \\
\hline grad01grad14 & -0.26 & -0.41 & -0.20 & grad06grad14 & -0.22 & -0.41 & -0.19 \\
\hline grad01grad15 & -0.26 & -0.44 & -0.21 & grad06grad15 & -0.22 & -0.44 & -0.19 \\
\hline $\operatorname{grad} 02 \operatorname{grad} 02$ & 0.00 & 0.00 & 0.00 & $\operatorname{grad} 07 \operatorname{grad} 07$ & -0.19 & -0.18 & -0.13 \\
\hline grad02grad03 & 0.00 & -0.13 & -0.08 & grad07grad08 & -0.19 & -0.15 & -0.11 \\
\hline grad02grad04 & 0.00 & -0.13 & -0.05 & grad07grad09 & -0.19 & -0.07 & -0.09 \\
\hline grad02grad05 & 0.00 & 0.00 & 0.00 & $\operatorname{grad} 07 \operatorname{grad} 10$ & -0.19 & -0.04 & -0.07 \\
\hline grad02grad06 & 0.00 & -0.22 & -0.12 & grad07grad11 & -0.19 & -0.30 & -0.15 \\
\hline grad02grad07 & 0.00 & -0.18 & -0.11 & $\operatorname{grad} 07 \operatorname{grad} 12$ & -0.19 & -0.33 & -0.16 \\
\hline grad02grad08 & 0.00 & -0.15 & -0.09 & grad07grad13 & -0.19 & -0.37 & -0.17 \\
\hline grad02grad09 & 0.00 & -0.07 & -0.06 & $\operatorname{grad} 07 \operatorname{grad} 14$ & -0.19 & -0.41 & -0.17 \\
\hline $\operatorname{grad} 02 \operatorname{grad} 10$ & 0.00 & -0.04 & -0.03 & $\operatorname{grad} 07 \operatorname{grad} 15$ & -0.19 & -0.44 & -0.18 \\
\hline grad02grad11 & 0.00 & -0.30 & -0.14 & grad08grad08 & -0.15 & -0.15 & -0.10 \\
\hline $\operatorname{grad} 02 \operatorname{grad} 12$ & 0.00 & -0.33 & -0.15 & grad08grad09 & -0.15 & -0.07 & -0.08 \\
\hline $\operatorname{grad} 02 \operatorname{grad} 13$ & 0.00 & -0.37 & -0.16 & $\operatorname{grad} 08 \operatorname{grad} 10$ & -0.15 & -0.04 & -0.06 \\
\hline grad02grad14 & 0.00 & -0.41 & -0.17 & grad08grad11 & -0.15 & -0.30 & -0.14 \\
\hline grad02grad15 & 0.00 & -0.44 & -0.17 & grad08grad12 & -0.15 & -0.33 & -0.14 \\
\hline grad03grad03 & -0.13 & -0.13 & -0.09 & $\operatorname{grad} 08 \operatorname{grad} 13$ & -0.15 & -0.37 & -0.15 \\
\hline grad03grad04 & -0.13 & -0.13 & -0.07 & grad08grad14 & -0.15 & -0.41 & -0.16 \\
\hline grad03grad05 & -0.13 & 0.00 & -0.04 & grad08grad15 & -0.15 & -0.44 & -0.16 \\
\hline grad03grad06 & -0.13 & -0.22 & -0.11 & grad09grad09 & -0.07 & -0.07 & -0.05 \\
\hline $\operatorname{grad} 03 \operatorname{grad} 07$ & -0.13 & -0.18 & -0.11 & $\operatorname{grad} 09 \operatorname{grad} 10$ & -0.07 & -0.04 & -0.04 \\
\hline grad03grad08 & -0.13 & -0.15 & -0.09 & $\operatorname{grad} 02 \operatorname{grad} 11$ & -0.00 & -0.30 & -0.14 \\
\hline grad03grad09 & -0.13 & -0.07 & -0.07 & grad09grad12 & -0.07 & -0.33 & -0.11 \\
\hline $\operatorname{grad} 03 \operatorname{grad} 10$ & -0.13 & -0.04 & -0.06 & $\operatorname{grad} 09 \operatorname{grad} 13$ & -0.07 & -0.37 & -0.11 \\
\hline grad03grad11 & -0.13 & -0.30 & -0.13 & grad09grad14 & -0.07 & -0.41 & -0.12 \\
\hline $\operatorname{grad} 03 \operatorname{grad} 12$ & -0.13 & -0.33 & -0.14 & $\operatorname{grad} 09 \operatorname{grad} 15$ & -0.07 & -0.44 & -0.12 \\
\hline $\operatorname{grad} 03 \operatorname{grad} 13$ & -0.13 & -0.37 & -0.14 & $\operatorname{grad} 10 \operatorname{grad} 10$ & -0.04 & -0.04 & -0.03 \\
\hline $\operatorname{grad} 03 \operatorname{grad} 14$ & -0.13 & -0.41 & -0.15 & $\operatorname{grad} 10 \operatorname{grad} 11$ & -0.04 & -0.30 & -0.08 \\
\hline grad03grad15 & -0.13 & -0.44 & -0.15 & $\operatorname{grad} 10 \operatorname{grad} 12$ & -0.04 & -0.33 & -0.09 \\
\hline grad04grad04 & -0.13 & -0.13 & -0.09 & $\operatorname{grad} 10 \operatorname{grad} 13$ & -0.04 & -0.37 & -0.09 \\
\hline grad04grad05 & -0.13 & 0.00 & -0.04 & grad10grad14 & -0.04 & -0.41 & -0.10 \\
\hline grad04grad06 & -0.13 & -0.22 & -0.14 & grad10grad15 & -0.04 & -0.44 & -0.10 \\
\hline grad04grad07 & -0.13 & -0.18 & -0.13 & $\operatorname{grad} 11 \operatorname{grad} 11$ & -0.30 & -0.30 & -0.19 \\
\hline grad04grad08 & -0.13 & -0.15 & -0.11 & $\operatorname{grad} 11 \operatorname{grad} 12$ & -0.30 & -0.33 & -0.20 \\
\hline grad04grad09 & -0.13 & -0.07 & -0.08 & grad11 grad13 & -0.30 & -0.37 & -0.21 \\
\hline grad04grad10 & -0.13 & -0.04 & -0.06 & grad11 grad14 & -0.30 & -0.41 & -0.22 \\
\hline grad04grad11 & -0.13 & -0.30 & -0.16 & grad11 grad15 & -0.30 & -0.44 & -0.22 \\
\hline grad04grad12 & -0.13 & -0.33 & -0.17 & $\operatorname{grad} 12 \operatorname{grad} 12$ & -0.33 & -0.33 & -0.21 \\
\hline grad04grad13 & -0.13 & -0.37 & -0.18 & $\operatorname{grad} 12 \operatorname{grad} 13$ & -0.33 & -0.37 & -0.22 \\
\hline grad04grad14 & -0.13 & -0.41 & -0.19 & $\operatorname{grad} 12 \operatorname{grad} 14$ & -0.33 & -0.41 & -0.23 \\
\hline grad04grad15 & -0.13 & -0.44 & -0.20 & $\operatorname{grad} 12 \operatorname{grad} 15$ & -0.33 & -0.44 & -0.24 \\
\hline grad05grad05 & 0.00 & 0.00 & 0.00 & $\operatorname{grad} 13 \operatorname{grad} 13$ & -0.37 & -0.37 & -0.23 \\
\hline grad05grad06 & 0.00 & -0.22 & -0.10 & $\operatorname{grad} 13 \operatorname{grad} 14$ & -0.37 & -0.41 & -0.24 \\
\hline grad05grad07 & 0.00 & -0.18 & -0.09 & grad13grad15 & -0.37 & -0.44 & -0.25 \\
\hline grad05grad08 & 0.00 & -0.15 & -0.07 & grad14grad14 & -0.41 & -0.41 & -0.25 \\
\hline grad05grad09 & 0.00 & -0.07 & -0.05 & $\operatorname{grad} 14 \operatorname{grad} 15$ & -0.41 & -0.44 & -0.26 \\
\hline grad05grad10 & 0.00 & -0.04 & -0.03 & grad15grad15 & -0.44 & -0.44 & -0.27 \\
\hline
\end{tabular}

remnant. This variation in outcomes would thereby have the effect of increasing the scatter as galaxies grow more massive. This is similar to what has been observed (Ogando et al. 2005, but see
Annibali et al. 2007). It may also explain the relative lack of galaxies with no gradients among the massive ellipticals (e.g., Ogando et al. 2005; Annibali et al. 2007). If the gradients 


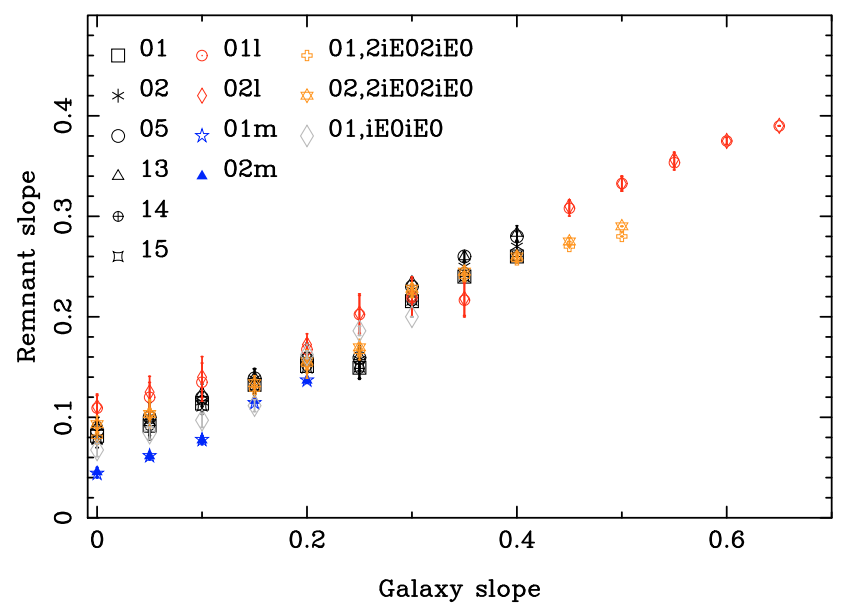

Fig. 5. Remnant (absolute) slopes as a function of the initial galaxy (absolute) slopes, for different orbits and different initial galaxy models. Symbols "01", "02", "05", "13", "14" and "15" in the legend correspond to interactions among two gE0 galaxies moving on different orbits (see Table 2 for the corresponding orbital parameters); "011" and " 021 " correspond to interactions among two gE01 galaxies with orbit id $=01$ and 02 , respectively; " $01 \mathrm{~m}$ " and " $02 \mathrm{~m}$ " correspond to interactions between two gE0m galaxies with orbit id $=01$ and 02 , respectively. Elliptical galaxies resulting from the merger of two $2 \mathrm{iE} 0$ galaxies are shown (with the symbols "01, 2iE02iE0" and "01, 2iE02iE0" referring to orbit id $=01$ and 02 , respectively). For comparison, also the slope resulting from the merger of two iE0 galaxies - having a total mass half of that of $\mathrm{gE} 0$ - are shown. Error bars correspond to the standard deviation of the mean.
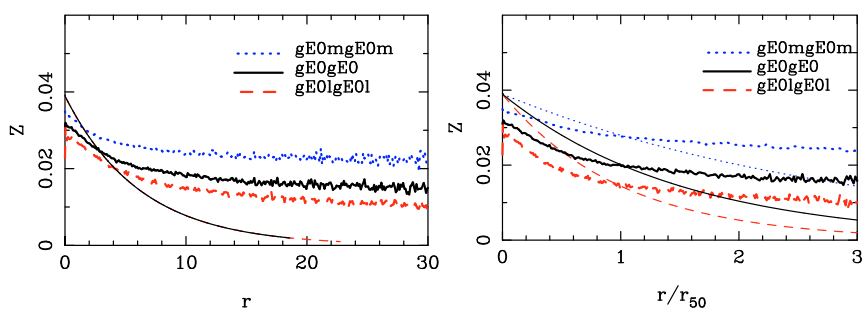

Fig. 6. Left panel: evolution of the metallicity profiles for three different major dry mergers, involving respectively two gE0 galaxies (black solid line), two gE01 galaxies (dashed red line) and two gE0m galaxies (dotted blue line). The initial profile is shown with corresponding thin curves. All the profiles are shown as a function of the distance $r$ form the galaxy center. Right panel: same as the previous panel, but this time all the profiles are shown in units of the half mass radius $r_{50}$ of the corresponding galaxy.

became flat, then they are likely to merge with galaxies that are steep enough to increase their slopes.

Note that, in this analysis, the metallicity gradients have been chosen to reflect those of elliptical galaxies in the local Universe. The progenitors of the most massive ellipticals observed today probably were low and intermediate mass ellipticals that merged at early epochs, and possibly had structural properties different from those of local galaxies. Unfortunately, the properties of distant early type galaxies are not well determined. This leaves no recourse but to use the observed properties of local galaxies. Thus we caution that our analysis may not capture in detail the cosmological evolution of elliptical galaxies.

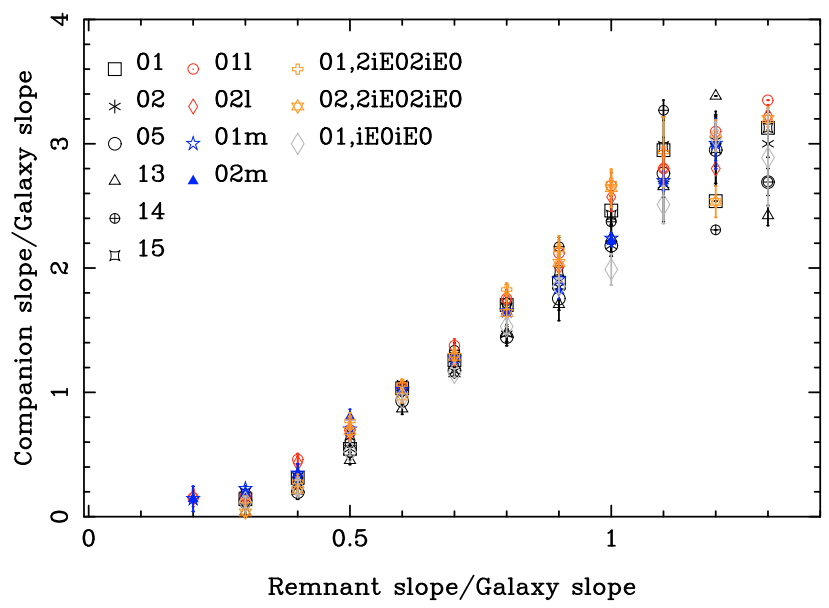

Fig. 7. Ratio of the companion (initial) slope to the galaxy (initial) slope versus the ratio of the remnant slope to the galaxy (initial) one. Symbols are described in caption of Fig. 5. Error bars correspond to the standard deviation of the mean. Note that the galaxies having a remnant slope of zero are not plotted in this figure. This corresponds to the situtation where the initial galaxy slope and companion slope are both zero and thus $1 /$ (galaxy slope) is infinite.

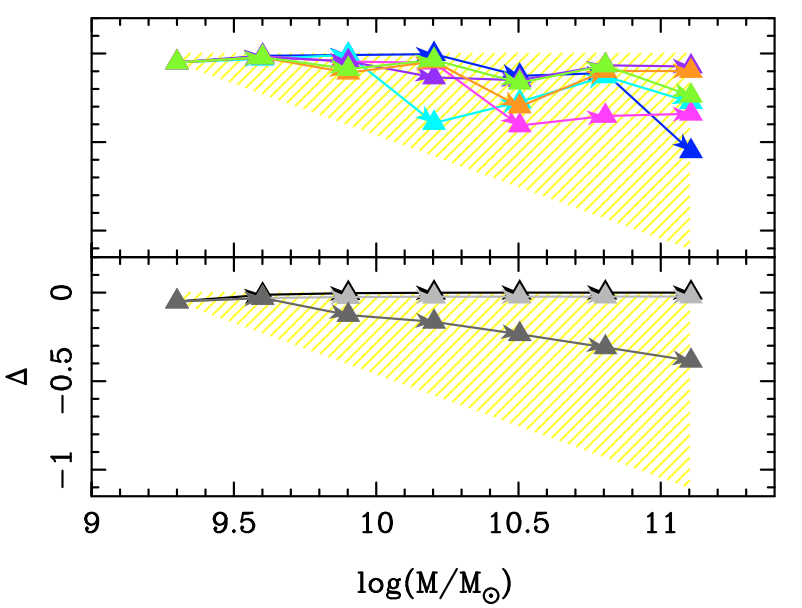

Fig. 8. Evolution of metallicity gradients through a sequence of major dry mergers. The initial elliptical galaxy has $\log \left(M / M_{\odot}\right)=9.3$ and $\Delta=-0.05$. (Upper panel) Different colors correspond to different merging histories, with companion gradients randomly distributed in the shaded area (cf. Fig. 2, right panel, in Ogando et al. 2005). (Lower panel) Different colors correspond to different merging histories, with companion gradients always equal to zero (black triangles), to the initial galaxy gradient (light gray triangles), and to the maximum slope allowed for a given mass, i.e., lower edge in the shaded area (dark gray triangles).

\section{Conclusions}

By means of a set of $N$-body simulations involving equal mass elliptical galaxies with no gas, we attempted to understand if dissipationless mergers always cause the flattening of the initial galaxy gradient, under what conditions does flattening happen, are there situations where a gradient could become steeper, what is the amount of flattening or steeping typically produced, and how does this evolution depend on orbital and morphological properties of the galaxies in these interacting systems. Our purpose is to explore how the metallicity gradients in dry mergers depends of the structure and metallicity gradients of the galaxies involved in the merger. Specifically, we would like to 
answer: Could dry mergers lead to metallicity gradients of the ellipticals observed in the local Universe?

From this modeling, we conclude that:

1. The ratio of the remnant and the initial galaxy slopes span a wide range of values, up to values greater than 1 (with values greater than one resulting only when companions have gradients twice the progenitor).

2. For a merger between two ellipticals having identical initial metallicity slope (i.e., companion and galaxy slopes being the same), the metallicity profile of the remnant flattens, with a final gradient about 0.6 times the initial one.

3. Ellipticals can maintain their original pre-merger metallicity gradient if the companion slope is sufficiently steep.

4. The final remnant gradient does not depend on the orbits of the progenitor ellipticals.

5. While the final gradient does depend on the initial concentration of the progenitors, the amount of flattening (i.e. remnant slope/progenitor slope) does not.

Given the diversity in outcomes of the mergers, we conclude that dry mergers do not violate any observational constraints on the systematic characteristics of metallicity gradients in local ellipticals. In fact, dry mergers might also explain many of the characteristics of metallicity gradients. There are no systematic trends in magnitude of metallicity gradients in ellipticals save one, metallicity gradients generally become steeper with increasing mass, but with an increasing dispersion (Ogando et al. 2005). This observation is logically explained within the context of our simulations: dry mergers lead to an overall decrease in the gradient, if the companion slope is not steep enough (companion slope/galaxy slope less than 2). We note however, that as the metallicity gradient gets flatter, it becomes easier for an elliptical to merge with another elliptical with sufficiently steep slope to actually increase the final metallicity gradient of the remnant. The reader should note that this mechanism requires the existence at any given time of progenitors with enough steep slopes, perhaps formed through a pure monolithic channel. Thus, if dry merging is important, we would expect the variance in the metallicity gradient to be the largest amongst the most massive elliptical consistent which is what is observed. Moreover, since steepening can occur, especially for ellipticals with no or shallow gradients, such a model also predicts that ellipticals will only rarely show very shallow gradients. This is something that has perhaps been observed (Ogando et al. 2005; Annibali et al. 2007) and is therefore an argument in favor of dry merging, not against it.

Acknowledgements. MDL wishes to thank the Centre National de la Recherche Scientifique (CNRS) for its continuing support of his research. P.D.M. is supported by a grant from the Agence Nationale de la Recherche (ANR) in France. The authors wish to thank the referee, for his prompt and constructive report.

\section{Appendix A: Mixing processes and their dipendence on the orbital parameters}

In Sect. 4.1 we have seen that the evolution of the metallicity profile for the fiducial merger between two gE0 galaxies does not depend on the energy and angular momentum of the orbit. As discussed in Sect. 2, the gE0 models represent quite idealized initial conditions, being spherical systems, without rotation. The final product of a merger of two not rotating spherical galaxies is typically a triaxial system, with some amount of rotation, due to the redistribution of the orbital angular momentum in internal one. Thus it can be interesting to understand if "hierarchical"
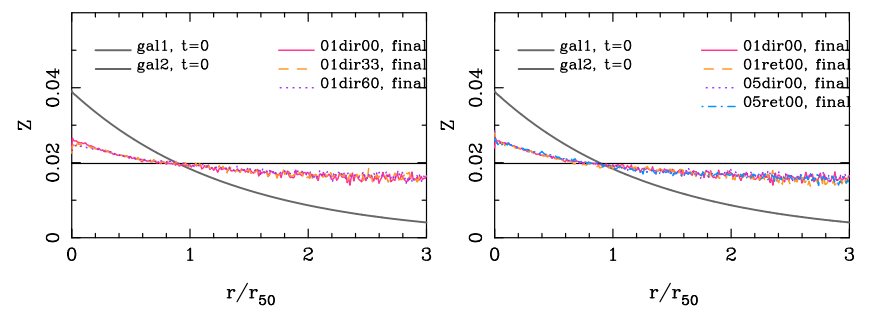

Fig. A.1. Evolution of the metallicity profiles in some major mergers involving two $2 \mathrm{iE0}$ galaxies. Metallicity is shown versus the distance from the galaxy center for the two galaxies before the interaction (black and thick gray solid lines) and for the final remnant for different orbits (see legends). The values on the abscissa are shown in units of the halfmass radius $r_{50}$ of the corresponding galaxy.
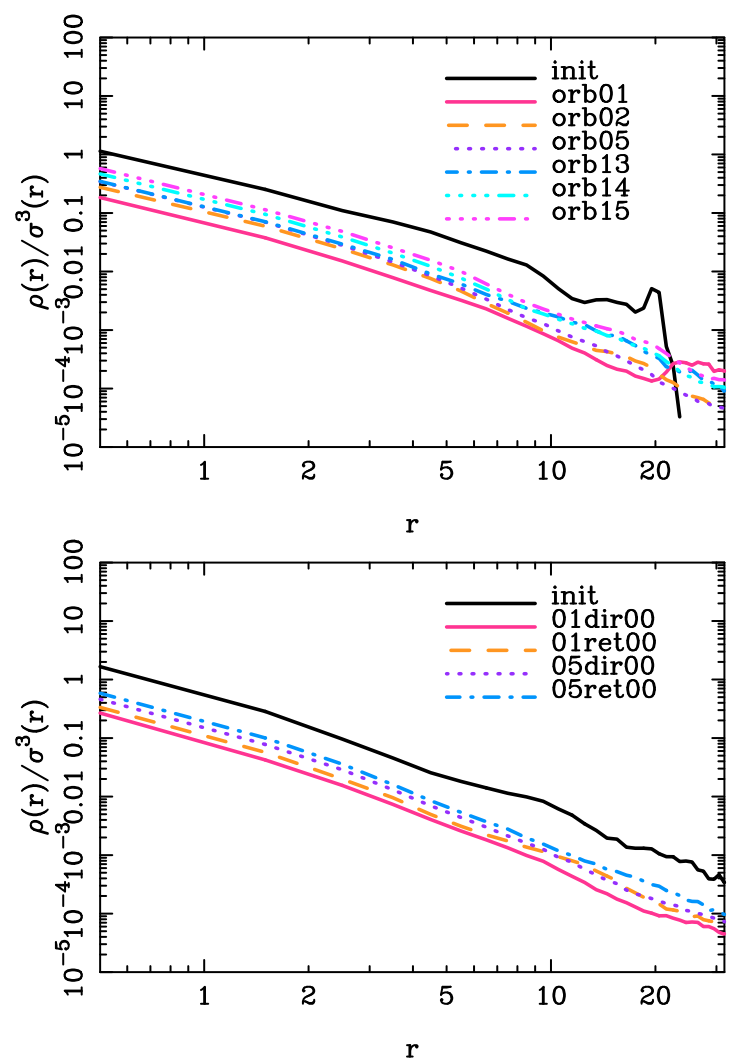

Fig. A.2. Evolution of the spherically averaged $Q(r)=\rho(r) / \sigma^{3}(r)$ during major dry mergers. Top panel: the profile $Q(r)$ is shown as a function of the distance $r$ (in kpc) from the galaxy center for the progenitor gE0 galaxy (solid thick black line) and for the remnant galaxies, for different orbits (see legend). Bottom panel: same as the previous panel, but this time the merger involves two $2 \mathrm{iE} 0$ galaxies, whose initial $Q(r)$ is shown by a black solid line. Different orientation between the orbital angular momentum and the galaxy spins have been taken into account in this case (direct and retograde orbits), as well as different orbital energies and angular momenta (orbit 01 and 05).

initial conditions (the initial galaxies being the remnants of previous mergers) still produce final metallicity profiles which show similar properties, independently on the parameters of the interaction. A different alignement between the initial galaxy spins and the orbital angular momentum can lead, for example, to different tidal effects in the interacting pairs. Can this also lead to final metallicity profiles depending on the initial orbital energy and angular momentum? In other words, are the results found in Sect. 4.1 confirmed when repeated hierarchical mergers are considered? 
To assess the generality of the results, we first simulated the merger of two iE0 galaxies (not rotating spherical galaxies whose masses are equal to half of the gE0 mass) and then two identical copies of the corresponding remnants were merged again, changing the initial orbital energy and angular momentum and its orientation with respect to the galaxy spins.

The result of these experiments are shown in Fig. A.1, where the metallicity profiles of $2 \mathrm{iE} 0-2 \mathrm{iE} 0$ mergers are shown, for different orbits, corresponding to different initial values of the orbital angular momentum (orbit id=01 and 05) and to different alignments of this orbital angular momentum with respect to the galaxy spins (dir00 and ret00 corresponding to the most extreme cases, when the orbital angular momentum is respectively parallel or antiparallel to the galaxy spins). In all the cases examined, it results that the final metallicity profiles are independent on the orbit of the encounter, at least inside the half mass radius of the remnant galaxy. It is interesting to note that this conclusion, i.e. the independence of the remnant metallicity profiles on the orbit of the encounter, does not depend on the morphological and kinematical properties of the initial systems either. Indeed it is valid when the merger involves two spherical symmetric, not rotating systems (Sect. 4.1), as well as when it involves two triaxial, rotating galaxies (Fig. A.1).

But where does this behaviour come from? In other words, how do mixing and phase-space density evolution proceed in collisionless major mergers and how do they depend on the orbital parameters of the encounter? The collisionless Boltzmann equation tells us that the evolution of a collisionless system is characterized by the conservation of the six-dimensional phasespace density (also known as the "fine-grained" phase-space density). The computation of this phase-space density being quite difficult, one usually computes the "coarse-grained" phasespace density, which represents the average of the fine-grained one over some finite volume in the phase-space, and which obeys one of the Mixing Theorems (see for example Tremaine et al. 1986) or the spherically averaged quantity $Q(r)=\rho(r) / \sigma^{3}(r)-$ being $\rho(r)$ the spherically averaged volume density and $\sigma(r)$ the velocity dispersion- which still has the dimensions of a phasespace density, but being only a proxy of the coarse-grained distribution function, it does not satisfy any of the Mixing Theorems.

Vass et al. (2008) have recently studied the evolution of the phase-space density distribution of dark matter halos during equal-mass mergers. They have taken into account a variety of shapes for the dark matter halos (from steep cusps to corelike profiles), of different orbital parameters, as well as recursive mergers of dark halos, in order to study the effect of a hierarchical building up of halos on the evolution of the phase-space density profiles. They studied both the evolution of the coarsegrained distribution function $F(r)$ and of its spherically averaged proxy $Q(r)$, showing that they evolve in a similar way during the merging process, independently on the orbital properties of the encounter and on the number of repeated collisions. In all cases, the inner slopes and the overall shapes of the phase-space density distribution of the merger remnant are close to that of the progenitor system.

We too found similar results evaluating the $Q(r)$ profile of our remnant galaxies, for different orbital initial conditions. As shown in Fig. A.2, mergers of spherically symmetric (upper panel) and of triaxial (lower panel) systems lead to an evolution of the spherically averaged $Q(r)$ which is independent on the orbit and which retains memory of the progenitor profile.

This independency in the evolution of the phase-space distribution on the orbital properties of the encounter leads also to very similar evolutions in the spatial distribution of particles

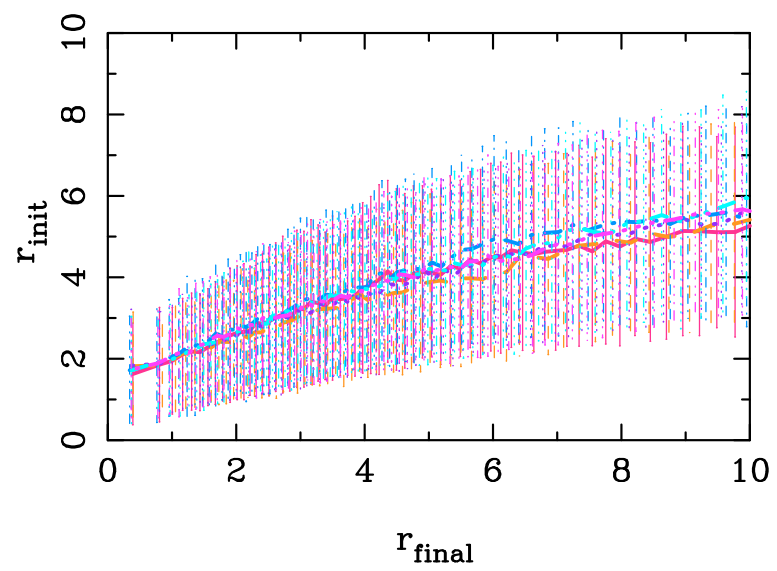

Fig. A.3. Redistribution of stellar particles in the final remnants of gE0gE0 mergers. For every stellar particle in the remnant, we have evaluated its position in the remnant (through its distance $r_{\text {final }}$ from the remnant center) and the position it had in the progenitor galaxy (through its distance $r_{\text {init }}$ from the progenitor center). The averaged quantities are then plotted, each curve corresponding to a different orbit, as explained in the legend of Fig. A.2, left panel. The corresponding standard deviations are also shown. Note that particles are redistributed in a very similar way in the merger remnants (similar averages and dispersions), independent of the orbit of the encounter.

after the collisions. In other words, stellar particles redistribute themselves in the remnant galaxy in a very similar way, independently on the orbits (see Fig. A.3), at least inside the remnant half-mass radius ( $r_{50} \simeq 7 \mathrm{kpc}$ for gE0-gE0 mergers). This ultimately gives rise to the very similar metallicity profiles found in Figs. 2 and A.1.

\section{References}

Annibali, F., Bressan, A., Rampazzo, R., Zeilinger, W. W., \& Danese, L. 2007, A\&A, 463, 455

Barnes J. E. 1996, in New Light on Galaxy Evolution, ed. R. Bender, \& R. L. Davies (Dordrecht: Kluwer), IAU Symp., 171, 191

Barnes, J., \& Hut, P. 1986, Nature, 324, 446

Bekki, K., \& Shioya, Y. 1999, ApJ, 513, 108

Bundy, K., Ellis, R. S., \& Conselice, C. J. 2005, ApJ, 625, 621

Carlberg, R. G. 1985, ApJ, 286, 403

Carlberg, R. G. 1986, ApJ, 310, 593

Carollo, C. M., Danziger, I. J., \& Buson, L. 1993, MNRAS, 265, 553

Cattaneo, A., \& Bernardi, M. 2003, MNRAS, 344, 45

Cattaneo, A., Dekel, A., Devriendt, J., Guiderdoni, B., \& Blaizot, J. 2006, MNRAS, 370, 1651

Cattaneo, A., Dekel, A., Faber, S. M., \& Guiderdoni, B. 2008, MNRAS, 389, 567

Chiosi, C., \& Carraro, G. 2002, MNRAS, 335, 335

Cimatti, A., Daddi, E., \& Renzini, A. 2006, A\&A, 453, 29

Ciotti, L., D’Ercole, A., Pellegrini, S., \& Renzini, A. 1991, ApJ, 376, 380

Clemens, M. S., Bressan, A., Nikolic, B., \& Rampazzo, R. 2009, MNRAS, 392, 35

Davies, R. L., Sadler, E. M., \& Peletier, R. F. 1993, MNRAS, 262, 650

De Lucia, G., Springel, V., White, S. D. M., Croton, D., \& Kauffmann, G. 2006, MNRAS, 366, 499

Di Matteo, P., Combes, F., Melchior, A.-L., \& Semelin, B. 2007, A\&A, 468, 61 Di Matteo, P., Bournaud, F., Martig, M., et al. 2008, A\&A, 492, 31

Emsellem, E., Cappellari, M., Krajnović, D., et al. 2007, MNRAS, 379, 401

Forbes, D. A., Sanchez-Blazquez, P., \& Proctor, R. 2005, MNRAS, 361, 6

Forbes, D. A., Strader, J., Brodie, J., \& Proctor, R. 2007

[arXiv: astro-ph/0702572]

Gingold, R. A., \& Monaghan, J. J. 1982, JCoPh, 46, 429

Hasinger, G., Miyaji, T., \& Schmidt, M. 2005, A\&A, 441, 417

Kawata, D. 1999, PASJ, 51, 931

Ko, J., \& Im, M. 2005, JKAS, 38, 149 
Kobayashi, C. 2004, MNRAS, 347, 740

Kobayashi, C., \& Arimoto, N. 1999, ApJ, 527, 573

Khochfar, S., \& Burkert, A. 2003, ApJ, 597, L117

Khochfar, S., \& Silk, A. 2006, ApJ, 648, L21

Larson, R. B. 1974, MNRAS, 166, 585

Lucy, L. B. 1977, AJ, 82, 1013

Matteucci, F. 1994, A\&A, 288, 57

Mihos, C., \& Hernquist, L. 1994, ApJ, 427, 112

Naab, T., Khochfar, S., \& Burkert, A. 2006, ApJ, 636, L81

Nelan, J. E., Smith, R. J., Hudson, M. J., et al. 2005, ApJ, 632, 137

Ogando, R. L. C., Maia, M. A. G., Chiappini, C., et al. 2005, ApJ, 632, 61

Pipino, A., \& Matteucci, F. 2008, A\&A, 486, 763

Pipino, A., D’Ercole, A., \& Matteucci, F. 2008, A\&A, 484, 679

Pipino, A., et al. 2009, in preparation

Sánchez-Blázquez, P., Gorgas, J., \& Cardiel, N. 2006, A\&A, 457, 823
Sánchez-Blázquez, P., Forbes, D. A., Strader, J., Brodie, J., \& Proctor, R. 2007, MNRAS, 377, 759

Semelin, B., \& Combes, F. 2002, A\&A, 388, 826

Spolaor, M., Proctor, R. N., Forbes, D. A., \& Couch, W. J. 2009, ApJ, 691, L138

Thomas, D., Maraston, C., Bender, R., \& Mendes de Oliveira, C. 2005, ApJ, 621, 673

Tremaine, S., Henon, M., \& Lynden-Bell, D. 1986, MNRAS, 219, 285

Valluri, M., Vass, I. M., Kazantzidis, S., Kravtsov, A. V., \& Bohn, C. L. 2007, ApJ, 658, 731

Vass, I. M., Kazantzidis, S., Valluri, M., \& Kravtsov, A. V. 2008, ApJ, submitted, [arXiv: 0812.3659]

van Albada, T. S. 1982, MNRAS, 201, 939

van Dokkum, P. G., Franx, M., Kriek, M., et al. 2008, ApJ, 677, L5

White, S. D. M. 1980, MNRAS, 191, 1

Worthey, G., Faber, S. M., \& Gonzalez, J. J. 1992, ApJ, 398, 69 